\title{
Interactions between star polymers: High-order calculations of the scaling exponents
}

\author{
V.Schulte-Frohlinde ${ }^{1}$, Yu.Holovatch ${ }^{2,3}$, C. von Ferber ${ }^{1}$, \\ A.Blumen ${ }^{1}$ \\ 1 Theoretische Polymerphysik, Universität Freiburg, \\ Hermann-Herder-Strasse 3, D-79104 Freiburg, Germany \\ 2 Institute for Condensed Matter Physics \\ of the National Academy of Sciences of Ukraine, \\ 1 Svientsitskii Str., 79011 Lviv, Ukraine \\ 3 Ivan Franko National University of Lviv, \\ 12 Drahomanov Str., 79005 Lviv, Ukraine
}

Received September 23, 2003

\begin{abstract}
The scaling behavior of star polymers can be calculated in the $m \rightarrow 0$ limit of an $m$-component spin system with an additional composite operator. The resulting scaling exponents describe the effective interaction of such polymer stars, i.e. objects whose behavior interpolates between that of polymer coils and that of hard sphere colloidal particles. We extend the existing renormalization group calculations from the third to the fourth order.
\end{abstract}

Key words: polymers, renormalization-group, fractals, critical exponents

PACS: $64.60 . A k, 61.41 .+e, 64.60 . F r, 11.10 . G h$

Star polymers which consist of linear polymer chains linked at one end are the simplest branched objects. They act as colloidal particles [1,2]; when increasing the number of arms their behavior changes from that of linear polymers to that of polymeric micelles [3]. From the scaling properties of star polymers, one may derive their effective interactions. The partition function of two star polymers with $f$ arms each and a distance $r$ between their cores obeys a power law governed by the so-called contact exponent $\Theta_{f f}[4]$

$$
\mathcal{Z}_{f f}^{(2)}(r) \sim r^{\Theta f}
$$

The logarithm of the partition function is the free energy, $\mathcal{F}_{f f}^{(2)}=-k_{\mathrm{B}} T \ln \mathcal{Z}_{f f}^{(2)}$. Differentiating $\mathcal{F}_{f f}^{(2)}$ with respect to $r$ gives the mean force $F_{f}(r)$ between two star 
polymers $[4-6]$ :

$$
\frac{1}{k_{\mathrm{B}} T} F_{f}(r)=\frac{\Theta_{f f}}{r}
$$

The mean force between the cores of two star polymers is experimentally accessible and determines the properties of star polymer solutions. Estimates for the dependence of the contact exponent $\Theta_{f f}$ on the number of arms $f$ have been found by scaling arguments and by using the so-called cone approximation $[7,8]$, giving $\Theta_{f f} \sim f^{3 / 2}$. Making use of the known results [5] for $\Theta_{f f}$ for $f=1$ and $f=2$ also fixes the prefactor. Thus [1]

$$
\Theta_{f f} \approx \frac{5}{18} f^{3 / 2}
$$

The contact exponents $\Theta_{f f}$ are related to a family of exponents for single star polymers $[4,9,10]$. We denote by $\mathcal{Z}^{(* f)}$ the partition function of a single star polymer with $f$ chains, each consisting of $N$ monomers. $\mathcal{Z}^{(* f)}$ obeys the following scaling form $[4,11]$ :

$$
\mathcal{Z}^{(* f)} \sim \mathrm{e}^{\mu N f} N^{\gamma_{f}-1}
$$

with the star configuration exponent $\gamma_{f}$ and a connectivity constant $\mathrm{e}^{\mu}$. We note that the exponents $\gamma_{f}$ are directly accessible through Monte Carlo (MC) simulations [1214]. In particular, $\gamma_{2}=\gamma_{1}$ is the configuration exponent of a polymer chain.

Now, the $\gamma_{f}$ can be expressed in terms of additional exponents $\eta_{f}$ which appear naturally in the field theory that we develop below. The relations between these exponents are [15]

$$
\gamma_{1}=\gamma_{2}=1-\nu \eta_{2}
$$

and

$$
\gamma_{f}=1+\nu\left(\eta_{f}-f \eta_{2}\right), \quad f>2 .
$$

In equations (5) and (6) $\nu$ is the usual Flory exponent, which relates the mean square end-to-end distance $R^{2}$ of the polymer chain to its number of monomers $N$ through $R^{2} \sim N^{2 \nu}$.

The star exponents $\gamma_{f}$ and $\eta_{f}$ are universal; they depend on $f$ and on the space dimension $d$ only [15]. The exponents $\eta_{f}$ allow to express $\Theta_{f f}$ in a simple way $[4,16]$ :

$$
\Theta_{f f^{\prime}}=\eta_{f}+\eta_{f^{\prime}}-\eta_{f+f^{\prime}}
$$

So far, $\eta_{f}$ (or equivalently $\gamma_{f}$ ) were evaluated perturbatively in expansions up to the third order both in $\varepsilon=4-d[4,8-11,15,17]$ and in fixed dimension $d=3$ [16]. Here, we calculate $\eta_{f}$ up to the order $\varepsilon^{4}$.

It is well known that the scaling exponents of polymer chains may generally be obtained from the $m \rightarrow 0$ limit of $O(m)$-symmetric $m$-vector magnets [18-20]. The same is true for the star exponents, but the underlying field theory has to be extended to represent stars. Our calculations are based on the field-theoretical renormalization group ( $R G$ ) approach in the minimal subtraction scheme [20-22]. We follow the formalism of [16] and refer the reader to [23] for a more detailed account. The field-theoretical description of a star polymer may be performed in 
terms of the Edwards model of continuous chains [24], generalized in order to describe a set of $f$ chains. The configuration of a linear polymer, say $a$, is given by a path $\mathbf{r}_{a}(s)$ in $d$-dimensional space $\mathbb{R}^{d}$ parameterized by a variable $0 \leqslant s \leqslant S_{a}$ where $S_{a}$ is given by $N l^{2}, l$ being the length of a monomer. Denoting the excluded volume interaction by $u$, the Hamiltonian $\mathcal{H}$ for $f$ chains is given by [15]

$$
\frac{1}{k_{\mathrm{B}} T} \mathcal{H}\left(\mathbf{r}_{a},\left\{S_{s}\right\}\right)=\sum_{a=1}^{f} \int_{0}^{S_{a}} \mathrm{~d} s\left(\frac{\mathrm{d} \mathbf{r}_{a}(s)}{2 \mathrm{~d} s}\right)^{2}+\frac{1}{6} \sum_{a, b=1}^{f} u \int \mathrm{d} \mathbf{r} \rho_{a}(\mathbf{r}) \rho_{b}(\mathbf{r}),
$$

with the densities $\rho_{a}(\mathbf{r})=\int_{0}^{S_{a}} \mathrm{~d} s \delta^{d}\left(\mathbf{r}-\mathbf{r}_{a}(s)\right)$. In this formalism the partition function of $f$ polymer chains is calculated as a functional integral:

$$
\mathcal{Z}^{(f)}\left\{S_{a}\right\}=\int \mathcal{D}\left[\mathbf{r}_{a}(s)\right] \exp \left\{-\frac{1}{k_{\mathrm{B}} T} \mathcal{H}\left(\mathbf{r}_{a},\left\{S_{a}\right\}\right)\right\} .
$$

Here, the symbol $\mathcal{D}\left[\mathbf{r}_{a}(s)\right]$ includes the normalization such that $\mathcal{Z}^{(* f)}\left\{S_{a}\right\}=1$ if $u \equiv 0$. In order to have a well-defined bare theory a cutoff $s_{0}$ is introduced such that all simultaneous integrals of any variables $s$ and $s^{\prime}$ on the same chain are cut off by $\left|s-s^{\prime}\right|>s_{0}$. The $f$ polymers can now be used to form a star by constraining them to have a common starting point. The corresponding partition function is:

$$
\mathcal{Z}^{(* f)}\left\{S_{a}\right\}=\int \mathcal{D}\left[\mathbf{r}_{a}\right] \exp \left\{-\frac{1}{k_{\mathrm{B}} T} \mathcal{H}\left(\mathbf{r}_{a},\left\{S_{a}\right\}\right)\right\} \prod_{a=2}^{f} \delta^{d}\left(\mathbf{r}_{a}(0)-\mathbf{r}_{1}(0)\right) .
$$

The continuous chain model of equations (9) and (10) can be mapped onto a corresponding field theory by a Laplace transform from the variables $S_{a}$ to the conjugate chemical potentials ("mass variables") $\mu_{a}[15]$ :

$$
\tilde{\mathcal{Z}}^{(* f)}\left\{\mu_{a}\right\}=\int_{0}^{\infty}\left(\prod_{b} \mathrm{~d} S_{b} \mathrm{e}^{-\mu_{b} S_{b}}\right) \mathcal{Z}^{(* f)}\left\{S_{a}\right\} .
$$

The Laplace-transformed partition function $\tilde{\mathcal{Z}}^{(* f)}\left\{\mu_{a}\right\}$ is expressed as the $m=0$ limit of the functional integral over vector fields $\phi_{a}, a=1, \ldots, f$ with $m$ components $\phi_{a}^{\alpha}, \alpha=1, \ldots, m$ :

$$
\tilde{\mathcal{Z}}^{(* f)}\left\{\mu_{b}\right\}=\left.\int \mathcal{D}\left[\phi_{a}(\mathbf{r})\right] \exp \left[-\mathcal{L}\left\{\phi_{b}, \mu_{b}\right\}\right]\right|_{m=0} .
$$

The Landau-Ginzburg-Wilson Lagrangian $\mathcal{L}$ of $f$ interacting fields $\phi_{b}$, each with $m$ components, reads

$$
\mathcal{L}\left\{\phi_{b}, \mu_{b}\right\}=\frac{1}{2} \sum_{a=1}^{f} \int \mathrm{d} \mathbf{r}\left[\mu_{a} \phi_{a}^{2}+\left(\nabla \phi_{a}(\mathbf{r})\right)^{2}\right]+\frac{1}{4 !} \sum_{a, a^{\prime}=1}^{f} u \int \mathrm{d} \mathbf{r} \phi_{a}^{2}(\mathbf{r}) \phi_{a^{\prime}}^{2}(\mathbf{r}),
$$

where $\phi_{a}^{2}=\sum_{\alpha=1}^{m}\left(\phi_{a}^{\alpha}\right)^{2}$. The limit $m=0$ in equation (12) can be understood as a selection rule for the diagrams that contribute to the perturbation theory expansions. 
The one particle irreducible (1PI) vertex functions $\Gamma^{(n)}\left(\mathbf{q}_{i}\right)$ of this theory are defined by:

$$
\delta\left(\sum \mathbf{q}_{i}\right) \Gamma_{a_{1} \ldots a_{n}}^{(n)}\left(\mathbf{q}_{i}\right)=\int \mathrm{e}^{\mathrm{i} \mathbf{q}_{i} \cdot \mathbf{r}_{i}} \mathrm{~d} \mathbf{r}_{1} \ldots \mathrm{d} \mathbf{r}_{n}\left\langle\phi_{a_{1}}\left(\mathbf{r}_{1}\right) \ldots \phi_{a_{n}}\left(\mathbf{r}_{n}\right)\right\rangle_{1 \mathrm{PI}, m=0}^{\mathcal{L}} .
$$

The average $\langle\cdots\rangle$ in equation (14) is taken with respect to the Lagrangian in equation (13), keeping only the contributions which correspond to one-particle-irreducible graphs and which have non-vanishing tensor factors in the limit $m=0$.

The vertex part of the Laplace transform of equation (10) is given by [15]:

$$
\begin{aligned}
& \delta\left(\mathbf{p}+\sum \mathbf{q}_{i}\right) \Gamma^{(* f)}\left(\mathbf{p}, \mathbf{q}_{1} \ldots \mathbf{q}_{f}\right)= \\
& =\int \mathrm{e}^{\mathrm{i}\left(\mathbf{p} \cdot \mathbf{r}_{0}+\mathbf{q}_{i} \cdot \mathbf{r}_{i}\right)} \mathrm{d} \mathbf{r}_{0} \mathrm{~d} \mathbf{r}_{1} \ldots \mathrm{d} \mathbf{r}_{f}\left\langle\phi_{1}\left(\mathbf{r}_{0}\right) \ldots \phi_{f}\left(\mathbf{r}_{0}\right) \phi_{1}\left(\mathbf{r}_{1}\right) \ldots \phi_{f}\left(\mathbf{r}_{f}\right)\right\rangle_{1 \mathrm{PI}, m=0}^{\mathcal{L}} .
\end{aligned}
$$

Thus, one obtains the vertex function $\Gamma^{(* f)}$ by insertion of the composite operator $\prod_{a} \phi_{a}$ into the 1PI vertex function with $f$ external legs. The scaling dimension of this operator defines the star exponent of the corresponding polymer.

Now, we sketch the RG analysis of the field theory in equation (13). The initial expressions for the calculations are the bare vertex functions $\left(\partial / \partial k^{2}\right) \Gamma^{(2)}(u), \Gamma^{(4)}(u)$, and $\Gamma^{(* f)}(u)$. Ultraviolet divergences occur when the bare vertex functions are evaluated naively $[20,22]$. The polymer limit $m=0$ leads to essential simplifications of the renormalization: each field $\phi_{a}$ and mass $\mu_{a}$ renormalizes as if the other fields were absent. Since the theory is renormalizable, we can collect all the divergences in the so-called renormalization factors $Z$ and define a finite theory of the same structure as the original one by renormalizing the parameters. Here we use dimensional regularization in which divergences are expressed as poles in $\varepsilon$, defined by $d=4-\varepsilon$.

We render the theory finite by using the minimal subtraction scheme in which only pole terms in $\varepsilon$ are subtracted. The renormalization is carried out with the help of the $\mathcal{K} \bar{R}$-operation [22], which recursively subtracts all pole terms of lower order. The expressions for the $Z$-factors are given as power series in the renormalized coupling constant $g$ :

$$
Z_{\phi_{a}}(g)=\mathcal{K} \bar{R} \Gamma_{a a}^{(2)}(g), \quad Z_{g}(g)=\mathcal{K} \bar{R} \Gamma^{(4)}(g),
$$

where the renormalized coupling $g$ is defined by:

$$
u=\kappa^{\varepsilon} Z_{\phi_{a}}^{-2} Z_{g} g \text {. }
$$

The parameter $\kappa$ sets the scale of the external momenta in the renormalization procedure.

In order to renormalize the star vertex functions the renormalization factors $Z_{* f}$ are introduced by:

$$
Z_{\phi_{a}}^{f / 2} Z_{* f}=\mathcal{K} \bar{R} \Gamma^{(* f)}(g) .
$$

The renormalized couplings $g$ and renormalizing $Z$-factors depend on the scale parameter $\kappa$. This dependence defines the RG functions by the following relations:

$$
\kappa \frac{\mathrm{d}}{\mathrm{d} \kappa} g=\beta(g), \quad \kappa \frac{\mathrm{d}}{\mathrm{d} \kappa} \ln Z_{\phi_{a}}=\eta_{\phi_{a}}(g), \quad \kappa \frac{\mathrm{d}}{\mathrm{d} \kappa} \ln Z_{* f}=\eta_{f}(g) .
$$



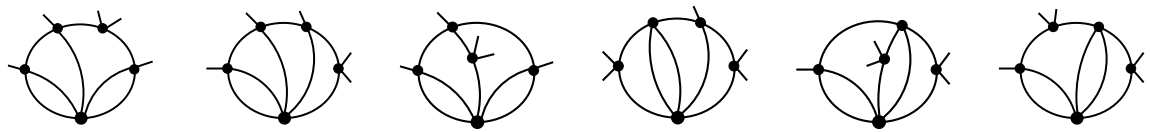

Figure 1. The contributing fourth order diagrams which have a $\phi^{5}$-vertex as star vertex.

The function $\eta_{\phi_{a}}(g)$ describes the pair correlation critical exponent [see equation (16)], while the functions $\eta_{f}(g)$ correspond to the set of exponents for polymer stars introduced in equation (5).

We have constructed explicit expressions for the $\beta$ and $\eta$ functions up to four loops. For the renormalized propagators and couplings of equations (16) and (17) we use the expansions for the corresponding magnetic spin system which are diagrammatic expansions with four-point interactions only, and apply the appropriate combinatorics in the limit $m \rightarrow 0$. For equation (18), we have to establish all terms containing an $f$-point interaction for the star vertex. At the four loop level this also includes a five-point interaction which does not appear among the four-point interactions in the spin system. Therefore, we have to calculate and renormalize six diagrams which contain a five-point interaction on top of their four-point interactions and which are shown in figure 1. It turns out that the integrations of five of these diagrams are identical to those of the diagrams which contain only four point interactions and which have been calculated for the spin system. We calculated the renormalized part of the last diagram in figure 1 by applying to it the so-called infrared rearrangement [25] and the $\bar{R}^{*}$-operation [26]. The details and explicit forms for the $\beta$ and $\gamma$-functions are given in a separate publication together with a study of the $\mathrm{RG}$ flow and the fixed points; there we have generalized the whole approach to cover the copolymer stars as well [23].

The asymptotic values of the scaling exponents were evaluated at the fixed point of the $\beta$-function. We find the following expansions for the polymer star exponents:

$$
\begin{aligned}
\eta_{f}= & f(f-1)\left\{-\frac{\varepsilon}{8}+\frac{\varepsilon^{2}}{256}[8(f-2)-9]+\varepsilon^{3}\left[a_{31}+(f-2)\left(a_{32}+(f-3) a_{33}\right)\right]\right. \\
& \left.+\varepsilon^{4}\left[a_{41}+(f-2)\left(a_{42}+(f-3)\left(a_{43}+(f-4) a_{44}\right)\right)\right]\right\} .
\end{aligned}
$$

Here

$$
\begin{aligned}
& a_{31}=33 \zeta_{3} / 512-49 / 4096 \\
& a_{32}=9 / 512-7 \zeta_{3} / 128 \\
& a_{33}=-1 / 64 \\
& a_{41}=477 \zeta_{3} / 16384+\left(99 \zeta_{4}-465 \zeta_{5}\right) / 2048+47 / 262144 \\
& a_{42}=\left(95 \zeta_{5}-21 \zeta_{4}\right) / 512-153 \zeta_{3} / 4096+133 / 32768 \\
& a_{43}=\left(65 \zeta_{3}+75 \zeta_{5}+1\right) / 2048 \\
& a_{44}=21 / 2048
\end{aligned}
$$

and $\zeta_{k}=\zeta(k)$ are the values of the Riemann- $\zeta$-function. Equation (20) recovers the $\varepsilon^{3}$ results of $[15,16]$. 


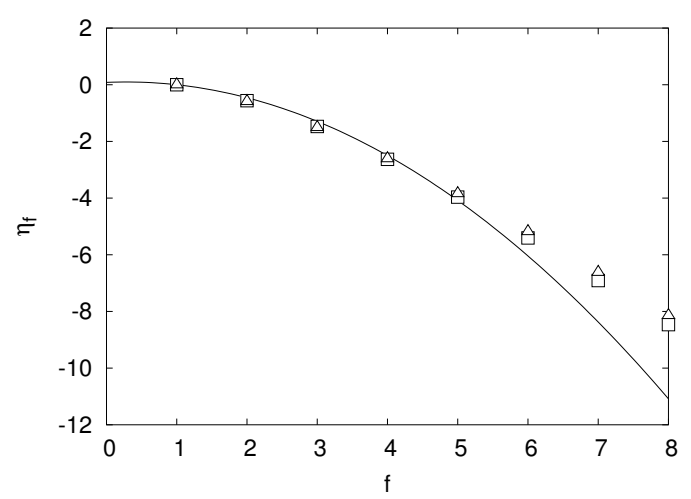

(a)

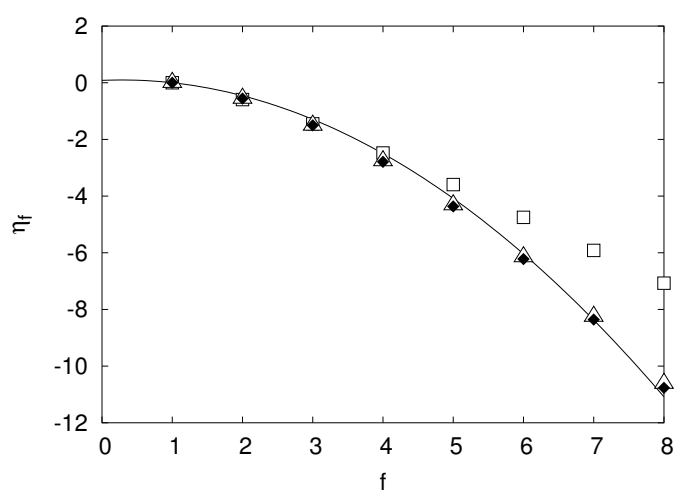

(b)

Figure 2. Star exponent $\eta_{f}$ for $d=2$. Lines: exact result [9]. (a) Conformal resummation; open squares: fourth order $\left(\varepsilon^{4}\right)$, open triangles: third order $\left(\varepsilon^{3}\right)$, (b) Padé approximants; open squares: $\varepsilon^{4}$ - Padé(2,2) approximant, open triangles: $\varepsilon^{3}$ - Padé(2,1) approximant, full diamonds: $\varepsilon^{4}$ - Padé(3,2) approximant.

As is well known, the RG perturbative expansions are asymptotic at best [27] and appropriate resummation techniques should be applied in order to extract reliable quantitative information from them [20,22]. Here, we apply a resummation procedure based on a conformal mapping, which is widely used in the analysis of perturbative RG expansions $[18,19]$. The procedure relies on the fact that the series for the $\beta$-functions of the $\phi^{4}$-theory are asymptotic and Borel-summable. In our case we assume that the perturbation series for $\eta_{f}$ is of the form

$$
\eta_{f}=\sum_{k \geqslant 1} A_{f, k}, \quad A_{f, k} \sim k !(\hat{a} f)^{k}, \quad k \gg 1,
$$

where $\hat{a}=3 / 8$ as found for the $\beta$-function [18]. For comparison we also apply simple Padé approximations. First, we report the results for the star exponents $\eta_{f}$. In figure 2 we give the third $\left(\sim \varepsilon^{3}\right)$ and fourth $\left(\sim \varepsilon^{4}\right)$ order results of the resummed $\varepsilon$-expansion for $d=2$, together with the exact result [9] for the exponent $\eta_{f}$, which reads:

$$
\eta_{f}(d=2)=-\frac{(f-1)(9 f+4)}{48} .
$$

Our results coincide with the exact result at small values of $f$. The expansion coefficients in the series for the star exponents grow with increasing $f$ and become, therefore, less reliable for larger values of $f$. Nonetheless, for all $f$ the $\varepsilon^{4}$ contribution is closer to the exact data than the third order approximation.

From figure 2(b), where we compare different Padé approximants for $\eta_{f}$ with the exact result, we conclude that better agreement is found for the non-diagonal approximants $(2,1)$ and $(3,2)$, which both have a quadratic high $f$ behavior. This seems to indicate that the weights of the contributions of the different $\varepsilon$-orders are not optimally chosen in our Borel resummation. A reason for this might be that the growth of the combinatorial factors in the expansion of the star exponents is stronger than assumed in equation (21). 


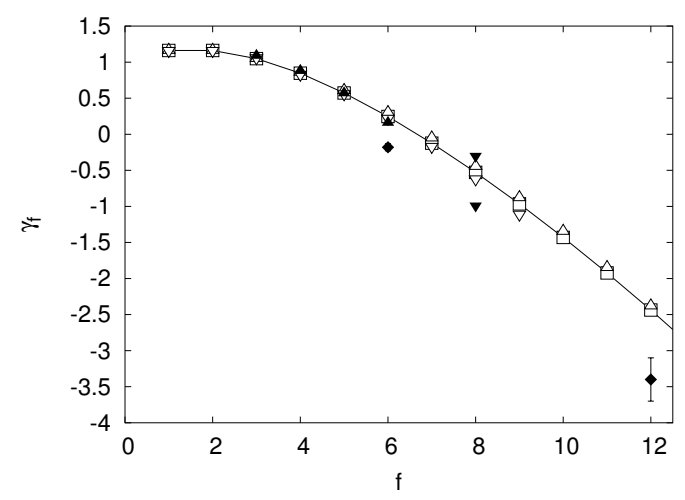

(a)

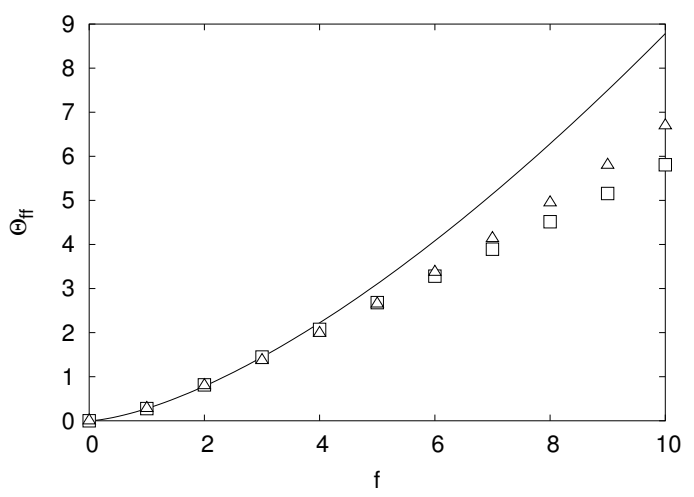

(b)

Figure 3. Star exponent $\gamma_{f}$ (a) and contact exponent $\Theta_{f f}(\mathrm{~b})$ at $d=3$. (a) Open squares: fourth order $\left(\varepsilon^{4}\right)$, open triangles up: third order $\left(\varepsilon^{3}\right)$, open triangles down: third order RG calculated at fixed dimension $(\mathrm{d}=3)$ [16], full triangles up: Monte Carlo simulations (MC) [13], full triangles down: MC [12], full diamonds: MC [14]. (b) Open squares: fourth order $\left(\varepsilon^{4}\right)$, open triangles: third order $\left(\varepsilon^{3}\right)$, line: cone approximation $\Theta_{f f}=\frac{5}{18} f^{3 / 2}$. The values for $\Theta_{f f}$ are found via equation (7) after resumming the series for the exponents $\eta_{f}$.

In figure 3(a) we compare the resummed $\varepsilon^{3}$ and $\varepsilon^{4}$ expansions for the $d=3$ star exponents. The exponents $\gamma_{f}$ were obtained from the $d=3$ data for $\eta_{f}$ [28] via equation (5), with $\nu=0.588$. These results are confronted with those of MC simulations $[12,13]$ and with the resummed three-loop pseudo- $\varepsilon$ expansion obtained within the massive $\mathrm{RG}$ scheme at fixed $d=3$ [16]. The agreement gets worse for larger $f$.

In figure 3(b) we plot the results for the exponent $\Theta_{f f}$, obtained through our method, which we compare to equation (3) established in the cone approximation. Two obvious conclusions from figure $3(\mathrm{~b})$ are: (i) for small $f$, where the perturbative expansions are known to give precise results, the cone approximation gives a reliable description; (ii) the fourth order expansion confirms the third order results in this respect.

In summary, we obtained in $d=2$ and $d=3$ the scaling exponents for a polymer star with an arbitrary number $f$ of arms. We confronted our results in $d=3$ with those of other approaches, based on Monte Carlo simulations [12,13,29], on the fixed dimension RG technique [16], and on the cone approximation [10]. In $d=2$, we compared our findings to the exact expressions [9]. Our perturbative approach to fourth order in $\varepsilon$ confirms the results previously obtained using lower order perturbation theory methods. A more careful study of the asymptotic behavior of our series might permit a refinement of the resummation in order to extract better numerical values for the star-star interaction at larger numbers $f$ of arms. 


\section{Acknowledgements}

We thank the Deutsche Forschungsgemeinschaft (SFB 428), the Fonds der Chemischen Industrie and the Bundesministerium für Bildung und Forschung (BMBF) for support.

\section{References}

1. Likos C.N., Löwen H., Watzlawek M., Abbas B., Jucknischke O., Allgaier J., Richter D. // Phys. Rev. Lett., 1998, vol. 80, p. 4450.

2. Watzlawek M., Likos C.N., Löwen H. // Phys. Rev. Lett., 1999, vol. 82, p. 5289; Jusufi A., Watzlawek M., Löwen H. // Macromolecules, 1999, vol. 32, p. 4470.

3. Seghtouchni R., Petekidis G., Fytas G., Semenov A.N., Roovers J., Fleischer G. // Europhys. Lett., 1998, vol. 42, p. 271.

4. Duplantier B. // J. Stat. Phys., 1989, vol. 54, p. 581.

5. des Cloizeaux J. // J. Phys. (Paris), 1980, vol. 41, p. 223.

6. von Ferber C., Holovatch Yu., Jusufi A., Likos C.N., Löwen H., Watzlawek M. // J. Mol. Liquids, 2001, vol. 93, p. 155.

7. Witten T.A., Pincus P.A. // Macromolecules, 1986, vol. 19, p. 2509; Europhys. Lett., 1986, vol. 2, p. 137.

8. Ohno K. // Phys. Rev. A, 1989, vol. 40, p. 1524.

9. Duplantier B. // Phys. Rev. Lett., 1986, vol. 57, p. 941; Saleur H. // J. Phys. A, 1986, vol. 19, p. L807; Duplantier B., Saleur H. // Phys. Rev. Lett., 1986, vol. 57, p. 3179.

10. Ohno K., Binder K. // J. Phys. (Paris), 1988, vol. 49, p. 1329.

11. Myake A., Freed K.F. // Macromolecules, 1983, vol. 16, p. 1228.

12. Barrett A.J., Tremain D.L. // Macromolecules, 1987, vol. 20, p. 1687.

13. Batoulis J., Kremer K. // Macromolecules, 1989, vol. 22, p. 4277.

14. Ohno K. // Macromol. Symp., 1994, vol. 81, p. 121.

15. Schäfer L., von Ferber C., Lehr U., Duplantier B. // Nucl. Phys. B, 1992, vol. 374, p. 473.

16. von Ferber C., Holovatch Yu. // Theor. Math. Phys. (Moscow), 1996, vol. 109, p. 34; Europhys. Lett., 1997, vol. 39, p. 31; Phys. Rev. E, 1997, vol. 56, p. 6370.

17. Wallace D.J., Zia R.K.P. // J. Phys. C, 1975, vol. 8, p. 839.

18. Le Guillou J.C., Zinn-Justin J. // Phys. Rev. B, 1980, vol. 21, p. 3976.

19. Guida R., Zinn-Justin J. // J. Phys. A, 1998, vol. 31, p. 8103.

20. Brezin E., Le Guillou J.C., Zinn-Justin J. - In: Phase Transitions and Critical Phenomena (edited by C.Domb, M.S.Green). New York, Academic Press, 1976, vol. 6, p. 125-247; Zinn-Justin J. Quantum Field Theory and Critical Phenomena (International Series of Monographs on Physics, 92). Oxford Univ. Press, 1996.

21. 't Hooft G., Veltman M. // Nucl. Phys. B, 1972, vol. 44, p. 189; 't Hooft G. // Nucl. Phys. B, 1973, vol. 61, p. 455.

22. Kleinert H., Schulte-Frohlinde V. Critical Properties of $\phi^{4}$-Theories. Singapore, World Scientific, 2001; Bogoliubov N.N., Shirkov D.V. Introduction to the Theory of Quantized Fields. New York, Wiley Interscience, 1958.

23. Schulte-Frohlinde V., Holovatch Yu., von Ferber C., Blumen A. (in preparation). 
24. des Cloizeaux J., Jannink G. Polymers in Solution. Oxford, Clarendon Press, 1990; Schäfer L. Universal Properties of Polymer Solutions as Explained by the Renormalization Group. Berlin, Springer, 1999.

25. Vladimirov A.A. // Theor. Math. Phys., 1978, vol. 36, p. 732.

26. Chetyrkin K.G., Tkachov F.V. // Phys. Lett. B, 1982, vol. 114, p. 340; Chetyrkin K.G., Smirnov V.A. // Phys. Lett. B, 1984, vol. 144, p. 419.

27. Hardy G.H. Divergent Series. Oxford, 1948.

28. Le Guillou J.C., Zinn-Justin J. // J. Physique, 1985, vol. 46, p. L137.

29. von Ferber C., Holovatch Yu. // Phys. Rev. E, 2002, vol. 65, p. 042801.

\section{Взаємодія між зірковими полімерами: обчислення скейлінгових показників у вищих порядках теорії збурень}

\section{В.Шулте-Фролінде ${ }^{1}$, Ю.Головач ${ }^{2,3}$, К. Фон Фербер ${ }^{1}$, А.Блюмен ${ }^{1}$}

1 Теоретична фізика полімерів, Університет Фрайбург, Німеччина, D-79104 Фрайбург, Герман-Гердер-Штрассе, 3

2 Інститут фізики конденсованих систем НАН України, 79011 Львів, вул. Свєнціцького, 1

3 Львівський національний університет ім. Івана Франка, 79005 Львів, вул. Драгоманова, 12

Отримано 23 вересня 2003 р.

Аналіз масштабної (скейлінгової) поведінки зіркових полімерів можна проводити на основі границі $m \rightarrow 0 \quad m$-компонентної спінової системи $з$ додатковим композитним оператором. Скейлінгові показники, які при цьому отримуються, описують ефективну взаємодію полімерних зірок: об'єктів, поведінка яких інтерполює між поведінкою полімерних клубків та колоїдних частинок. Ми доповнюємо існуючі ренормгрупові обчислення третього порядку теорії збурень четвертим.

Ключові слова: полімери, ренормалізаційна група, фрактали, критичні показники

PACS: $64.60 . A k, 61.41 .+e, 64.60 . F r, 11.10 . G h$ 
